\title{
Prediction of prolonged mechanical ventilation after cardiac surgery: An imperfect crystal ball
}

\author{
Juan N. Pulido, MD
}

\footnotetext{
From the Cardiovascular Intensive Care Unit, Cardiothoracic Anesthesiology and Critical Care Medicine, Swedish Heart and Vascular Institute, Physicians Anesthesia Services, Seattle, Wash. Disclosures: Author has nothing to disclose with regard to commercial support.

Received for publication Sept 8, 2016; accepted for publication Sept 8, 2016; available ahead of print Sept 30, 2016.

Address for reprints: Juan N. Pulido, MD, Cardiovascular Intensive Care Unit, Cardiothoracic Anesthesiology and Critical Care Medicine, Swedish Heart and Vascular Institute, Physicians Anesthesia Services, 550 17th Ave, 680, Seattle, WA 98122 (E-mail: juan.pulido@swedish.org).

J Thorac Cardiovasc Surg 2017;153:116-7

$0022-5223 / \$ 36.00$

Copyright (c) 2016 by The American Association for Thoracic Surgery

http://dx.doi.org/10.1016/j.jtcvs.2016.09.009
}

The clinical and financial impact of prolonged mechanical ventilation after cardiac surgery is well recognized and the subject of numerous initiatives in perioperative cardiac care. $^{1-3}$ Moreover, prolonged mechanical ventilation after coronary artery bypass grafting-defined as the percentage of patients who required intubation for longer than 24 hours after isolated coronary artery bypass grafting - is an important quality performance measure by the Society of Thoracic Surgeons. ${ }^{4}$ Having a comprehensive understanding of the factors that increase the risk of this complication can help providers, families, and patients to make the right decision, further optimize care when possible, and be prepared for the potential unwanted consequences of prolonged ventilation. Unfortunately, the crystal ball is not always clear, and not too infrequently both providers and families end up caught by surprise.

In their report in this issue of the Journal, Sharma and colleagues $^{5}$ have attempted to address this subject through the development of a prediction model of prolonged mechanical ventilation after cardiac surgery that was derived and validated in 2 very large retrospective cohorts. The study included adult patients undergoing cardiac surgery with cardiopulmonary bypass through a period of 12 years and defined prolonged mechanical ventilation as duration exceeding 48 hours. Instead of the standard 24 hours, Sharma and colleagues ${ }^{5}$ explicitly selected the target mechanical ventilation duration as a 48-hour threshold to overcome such factors as slow recovery from anesthesia and transient or minor complications, which in reality may not account for the true morbidity of this problem, and to avoid possible overestimation of mechanical ventilation's effects. After creation of an initial prediction model incorporating a dozen readily available perioperative variables, each model was validated on bootstrap replications to assess excessive optimism in regression coefficients and performance measures. The prevalences of prolonged mechanical ventilation were $6.2 \%$ and $7.3 \%$ in the derived and validation cohorts,

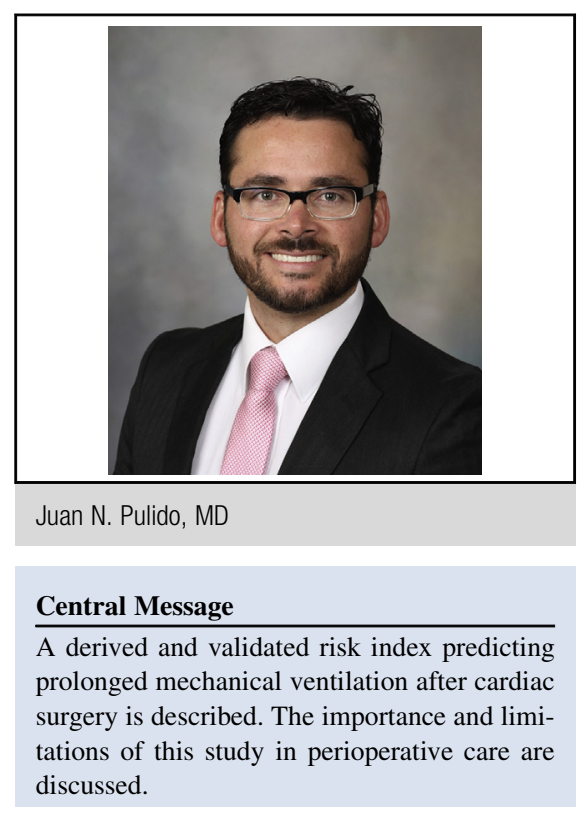

See Article page 108

respectively. Previous cardiac surgery, cardiogenic shock, and prolonged cardiopulmonary bypass time were identified as the major predictors. Although these may not come as surprise risk factors, they prove to be strong predictors after validation of a very large cohort and therefore should be considered seriously when receiving patients from the operating room and caring for them in the decisive initial postoperative phase of care.

Despite multiple studies on this subject, most data to date have been limited by sample size. Sharma and colleagues ${ }^{5}$ have taken this issue to another level with a very large, heterogeneous sample size and the addition of a validation cohort to affirm the weight of the risk factors. The importance if these findings exceed the mere enumeration and confirmation of predictors of prolonged ventilation after cardiac surgery. This risk index may help providers in deciding on optimal surgical timing and preoperative optimization as well as in risk stratification, thus aiding in postoperative resource management and intensive care unit use in treating cardiac surgical patients. Conversely, it can also help patients and families with informed consent and decision making before embarking on a challenging operation.

It is important, however, to acknowledge that when it comes to risk assessment there is no such thing as the 
perfect crystal ball. An important limitation of retrospective observational studies and risk prediction models is that their conclusions can only be applied to the variables collected.

How many patients who are seen with the mentioned high-risk variables go through complex operations under prolonged cardiopulmonary bypass and recover uneventfully with short postoperative ventilator needs? The answer is far too many for us to rely solely on predictors. Clinical acumen and experience have to complement these risk assessment tools to ensure that we continue to strive for optimal high-quality perioperative care.

The impact of fresh postoperative or "intensive care unit landing" hemodynamic and respiratory variables cannot be overemphasized and perhaps should be accounted for in future studies. These are the variables in the context of preoperative and intraoperative data that clinicians use to make decisions in postoperative care.

\section{References}

1. Hefner JL, Tripathi RS, Abel EE, Farneman M, Galloway J, Moffatt-Bruce SD Quality improvement intervention to decrease prolonged mechanical ventilation after coronary artery bypass surgery. Am J Crit Care. 2016;25:423-30.

2. Trouillet JL, Combes A, Vaissier E, Luyt CE, Ouattara A, Pavie A, et al. Prolonged mechanical ventilation after cardiac surgery: outcome and predictors. J Thorac Cardiovasc Surg. 2009;138:948-53.

3. Cohen AJ, Katz MG, Frenkel G, Medalion B, Geva D, Schachner A. Morbid results of prolonged intubation after coronary artery bypass surgery. Chest. 2000;118:1724-31.

4. The Society of Thoracic Surgeons. Quality performance measures. Available at: http://www.sts.org/quality-research-patient-safety/quality/quality-performancemeasures. Accessed September 2, 2016.

5. Sharma V, Rao V, Manlhiot C, Fremes S, Wasowicz M. A derived and validated score to predict prolonged mechanical ventilation in patients undergoing cardiac surgery. J Thorac Cardiovasc Surg. 2017;153:108-15. 